\title{
O uso da estratégia de alongamento compensatório em diferentes gravidades do desvio fonológico
}

\section{The use of the compensatory lengthening strategy in different severities of phonological disorder}

\author{
Brunah de Castro Brasil'1, Roberta Michelon Melo², Helena Bolli Mota ${ }^{3}$, Roberta Freitas Dias ${ }^{4}$, Carolina Lisbôa \\ Mezzomo ${ }^{5}$, Vanessa Giacchini ${ }^{6}$
}

\begin{abstract}
RESUMO
Objetivos: Verificar a existência de relação entre o uso da estratégia de alongamento compensatório e diferentes gravidades do desvio fonológico classificado quantitativa e qualitativamente. Além disso, verificar a existência de correspondência entre a gravidade do desvio fonológico nas duas classificações utilizadas no estudo. Métodos: Participaram da pesquisa 20 crianças com diagnóstico de desvio fonológico apresentando diferentes gravidades e com idades entre cinco e dez anos. Os desvios foram classificados de maneira quantitativa e qualitativa. O uso da estratégia estudada foi identificado com o auxílio da análise acústica, através do software de áudio-processamento (PRAAT). Os dados coletados foram analisados estatisticamente por meio do teste Qui-quadrado. Resultados: O estudo comparativo entre as gravidades do desvio fonológico, segundo as classificações quantitativa e qualitativa, não mostrou diferença estatisticamente significante entre os grupos no que se refere à estratégia de alongamento compensatório. Através da análise descritiva da distribuição dos sujeitos conforme a gravidade do desvio fonológico nas duas classificações, verificou-se correspondência entre as classificações quantitativa e qualitativa no que diz respeito à gravidade do desvio fonológico. Conclusão: Não houve relação entre o uso da estratégia de alongamento compensatório e as diferentes gravidades do desvio fonológico. A distribuição dos sujeitos por gravidade do desvio fonológico, segundo as classificações utilizadas, permitiu visualizar uma correspondência entre os dois tipos de classificações do desvio.
\end{abstract}

Descritores: Distúrbios da fala; Acústica da fala; Espectrografia do som/análise; Patologia da fala e linguagem; Linguagem infantil

\section{INTRODUÇÃO}

A aquisição fonológica considerada normal ocorre com o domínio do sistema fonológico padrão atingido espontanea-

Trabalho realizado no Curso de Fonoaudiologia da Universidade Federal de Santa Maria - UFSM - Santa Maria (RS), Brasil, com auxílio do Conselho Nacional de Desenvolvimento Científico e Tecnológico - CNPq, por meio do Programa Institucional de Bolsa de Iniciação Científica (PIBIC/UFSM). (1) Acadêmica do Curso de Fonoaudiologia da Universidade Federal de Santa Maria - UFSM - Santa Maria (RS), Brasil.

(2) Acadêmica do Curso de Fonoaudiologia da Universidade Federal de Santa Maria - UFSM - Santa Maria (RS), Brasil.

(3) Doutora, Professora do Curso de Fonoaudiologia e do Programa de PósGraduação em Distúrbios da Comunicação Humana da Universidade Federal de Santa Maria - UFSM - Santa Maria (RS), Brasil.

(4) Pós-graduanda (Mestrado) em Distúrbios da Comunicação Humana da Universidade Federal de Santa Maria - UFSM - Santa Maria (RS), Brasil.

(5) Doutora, Professora do Curso de Fonoaudiologia e do Programa de PósGraduação em Distúrbios da Comunicação Humana da Universidade Federal de Santa Maria - UFSM - Santa Maria (RS), Brasil.

(6) Pós-graduanda (Mestrado) em Distúrbios da Comunicação Humana pela Universidade Federal de Santa Maria - UFSM - Santa Maria (RS), Brasil. Endereço para correspondência: Helena Bolli Mota. R. José Carlos Kruel, 41/601, Santa Maria (RS), Brasil, CEP: 97060-380. E-mail: helenabolli@hotmail.com

Recebido em: 1/9/2008; Aceito em: 24/7/2009 mente, por volta dos cinco anos, em uma sequência comum à maior parte das crianças. Contudo, variações individuais podem ser percebidas entre elas, tanto no domínio segmental, quanto no domínio prosódico ${ }^{(1)}$.

No que se refere à estrutura silábica do Português Brasileiro (PB), o onset complexo (OC) é a última a ser adquirida no desenvolvimento fonológico normal (DFN), estabelecendo-se aos cinco anos. Esta estrutura silábica é composta por duas consoantes, sendo a primeira posição obrigatoriamente preenchida por uma obstruinte e a segunda por uma líquida, lateral (/l/) ou não-lateral (/r/), configurando a estrutura $\mathrm{C}^{1} \mathrm{C}^{2} \mathrm{~V}^{(2)}$.

Durante o período de aquisição fonológica, tanto normal, como desviante, a criança lança mão de estratégias de reparo no intuito de compensar suas dificuldades em produzir determinados segmentos e/ou estruturas silábicas e adequar seu sistema fonológico ao alvo-adulto ${ }^{(1,3)}$. As estratégias de reparo são superadas no decorrer do $\mathrm{DFN}^{(4)}$. No que se refere à aquisição do $\mathrm{OC}$, a estratégia de reparo frequentemente utilizada é a simplificação - produção $\mathrm{C}^{1} \mathrm{~V}$, sendo esta a última a desaparecer no curso do desenvolvimento fonológico ${ }^{(2,5-7)}$.

Algumas pesquisas mostram a estratégia de alongamento compensatório (AC) como um dos recursos utilizados na tentativa de produção do OC (prato - [pa:tu] $)^{(8)}$ e da coda $(\mathrm{CVC})$ 
(carta - [ka:ta $])^{(9-11)}$. Tanto as vogais como as consoantes fricativas da estrutura $\mathrm{C}^{1} \mathrm{C}^{2} \mathrm{~V}$ podem ser alongadas, como por exemplo, frio - [f:iu].

Os recursos empregados pelos sujeitos na tentativa de produzir o fonema-alvo não são aleatórios, podendo apresentar características próximas ao alvo e à estrutura silábica correspondente, o que remete a um possível conhecimento fonológico por parte da criança, o qual está em contínuo processo de desenvolvimento ${ }^{(11,12)}$.

Esse conceito é empregado à estratégia de $\mathrm{AC}$, pouco citada na literatura, e definida como sendo um fator compensatório para suprir a ausência de um segmento que a criança não consegue produzir (no caso da simplificação do OC é a líquida lateral ou não-lateral que compõem a estrutura silábica). Esta estratégia mostra um conhecimento linguístico por parte do falante na tentativa de manter a unidade temporal da sílaba ${ }^{(9,10)}$. A identificação deste tipo de estratégia é subsidiada pela análise acústica, uma vez que dificilmente é reconhecida apenas por meio da análise perceptivo-auditiva ${ }^{(10)}$.

Muitos dos erros apresentados por crianças com desvio fonológico (DF) são decorrentes de confusões ou falta de conhecimento, de uma ou mais características acústicas utilizadas na distinção dos sons da língua na qual a criança está inserida. Assim, a análise acústica proporciona uma base objetiva, bastante segura e conveniente, para a identificação e interpretação destes erros fonológicos ${ }^{(13-15)}$. Além disso, as medidas espectrográficas auxiliam na prática clínica, seja no momento do diagnóstico, na escolha do método terapêutico ou na investigação da efetividade da terapia fonoaudiológica. Podem, ainda, revelar ensaios e amostras de uma fonologia mais complexa do sujeito antes não revelada, vindo, portanto, complementar a análise perceptivo-auditiva ${ }^{(10)}$.

Quando a aquisição fonológica não segue uma sequência comum e espontânea apresentada pela maioria dos falantes de uma determinada língua, tem-se o que se denomina DF, caracterizado, também, pela inadequação do sistema fonológico do falante. A inadequação fonológica em alguns casos pode ser concomitante a dificuldades no nível fonético, com consequentes prejuízos na produção linguística. Nos DF não se observam problemas causais detectáveis, como dificuldade geral de aprendizagem, déficit intelectual, desordens neuromotoras, distúrbios psiquiátricos e/ou fatores ambientais ${ }^{(1,16,17)}$.

O DF pode ser classificado em diferentes gravidades, segundo critérios quantitativos ou qualitativos. A classificação quantitativa mais utilizada na clínica fonoaudiológica é o Percentual de Consoantes Corretas - Revisado (PCC-R ${ }^{(18)}$, que desconsidera as distorções produzidas pelo sujeito e é baseado no cálculo do Percentual de Consoantes Corretas (PCC) ${ }^{(19)}$. O PCC é calculado dividindo-se o número de consoantes produzidas corretamente pelo número de consoantes totais produzidas. Por fim, multiplica-se o valor encontrado por 100. A gravidade do DF nesta classificação é dividida em: média (DM), média-moderada (DMM), moderada-severa (DMS) e severa (DS). O DM tem PCC entre $86 \%$ e $100 \%$, o DMM PCC entre 66 e $85 \%$, o DMS PCC entre 51 e $65 \%$ e o DS PCC menor que $50 \%$.

No que se refere às classificações qualitativas, tem-se uma tipologia baseada nas características do sistema fonológico dos sujeitos, e também, na natureza do desvio deste sistema ${ }^{(20)}$. Nesta classificação, a gravidade do desvio pode ser de quatro tipos: DF com características atrasadas (CA), DF com características iniciais (CI), DF com características incomuns (CIc) e DF com características fonéticas adicionais (CFA).

Os sujeitos com CA apresentam sistemas fonológico e fonético com poucas trocas e/ou omissões, com alterações típicas de estágio final do desenvolvimento normal da linguagem (anteriorização de fricativa, apagamento de líquida não-lateral final, simplificação de OC), maioria dos processos fonológicos já superados e fala espontânea mais inteligível que os demais tipos. Os sujeitos que apresentam DF com CI caracterizam-se por um sistema fonológico típico de desenvolvimento inicial, presença de processos fonológicos iniciais (plosivização, dessonorização), restrição de contrastes de traços distintivos e ininteligibilidade na fala espontânea, porém, menos severa que no tipo com CIc. Este último apresenta sistemas fonológico e fonético restritos, processos fonológicos incomuns (fricatização, glotalização, apagamento de fricativa ou plosiva), presença de determinado som constantemente na fala espontânea, que se apresenta ininteligível, e restrição quanto aos contrastes de traços. Os sujeitos com CFA caracterizam-se por apresentar alterações fonéticas (relacionadas a frênulo lingual curto, otites frequentes, tonsila palatina hipertrófica, respiração oral, etc.) e características dos tipos de DF já citados.

Baseado nos dados da literatura ${ }^{(1,10,11)}$, para realização do presente estudo as autoras elaboraram a hipótese de que sujeitos com maior conhecimento fonológico, ou seja, com menor gravidade do DF, fariam uso mais frequente da estratégia de AC.

Este estudo teve como objetivo verificar a existência de relação entre o uso da estratégia de $\mathrm{AC}$ e diferentes gravidades do DF classificado quanti e qualitativamente. Além disso, verificar a existência de correspondência entre a gravidade do DF em duas classificações.

\section{MÉTODOS}

Os dados foram levantados a partir dos bancos de dados de dois projetos de pesquisa, aprovados pelo Comitê de Ética da Universidade Federal de Santa Maria (UFSM) (064/2004 e 23081.010335/2007-63).

Participaram 170 sujeitos, os quais foram submetidos à triagem fonoaudiológica, avaliando-se aspectos de linguagem, sistema estomatognático, voz e audição.

Para que os dados dos sujeitos fossem incluídos nesta pesquisa, os seguintes critérios foram considerados: apresentar diagnóstico de DF, realizar simplificação de OC, estarem autorizadas pelos pais ou responsáveis a participar da pesquisa por meio do Termo de Consentimento Livre e Esclarecido (TCLE) e terem idades entre cinco e dez anos.

Como critérios de exclusão consideraram-se os seguintes aspectos: sujeitos que tivessem recebido ou estivessem recebendo qualquer tipo de terapia fonoaudiológica, a não assinatura do TCLE, presença de alterações fonoaudiológicas além do desvio fonológico, e presença de comprometimentos evidentes nos aspectos neurológico, cognitivo e psicológico.

Atenderam aos critérios desta pesquisa 21 crianças com 
DF de diferentes gravidades, sendo dez do sexo feminino e 11 do masculino, com idades entre cinco e dez anos.

Foram coletadas amostras de fala, obtidas em ambiente silencioso e registradas com o uso do gravador digital Powerpack - Digital Voice Recorder DRV-800III. Os dados foram armazenados em um computador por meio do programa Recorder V2.0 Digital Voice ${ }^{\circledR}$. Os DF foram analisados e classificados utilizando-se a Avaliação Fonológica da Criança $(\mathrm{AFC})^{(16)}$. Todos os registros de fala foram transcritos por meio de transcrição fonética restrita e revistos por mais dois julgadores - acadêmicos do Curso de Fonoaudiologia da UFSM, com experiência em transcrição fonética - separadamente. As gravações foram analisadas e utilizadas para a classificação do DF e para a identificação do uso da estratégia de AC.

Foram utilizadas duas diferentes classificações para a gravidade do DF, sendo uma quantitativa e outra qualitativa.

Para a análise quantitativa do DF foi utilizado o PCC- $\mathrm{R}^{(18)}$, baseado na classificação do $\mathrm{PCC}^{(19)}$, que divide a gravidade do DF em DM, DMM, DMS e DS.

Através da análise dos dados da avaliação fonológica, utilizando a classificação quantitativa, verificou-se que oito sujeitos apresentavam DM; sete sujeitos DMM; cinco sujeitos DMS e um sujeito DS.

Para a análise qualitativa, foi utilizada uma tipologia ${ }^{(20)}$, na qual, conforme a gravidade, o DF pode ser: DF com CA, DF com CI, DF com CIc e DF com CFA.

$\mathrm{Na}$ análise dos dados da avaliação fonológica, utilizando a classificação qualitativa, verificou-se que 11 sujeitos apresentavam desvio com CA, nove com CI e um com CIc. Nenhum sujeito foi classificado como apresentando desvio com CFA.

Para a obtenção dos registros de fala submetidos à análise acústica foi elaborada uma lista contendo 40 pares de palavras contrastando em relação às estruturas $\mathrm{CCV}$ versus $\mathrm{CV}$ e CVC versus $\mathrm{CV}$ (ex.: frio x fio; carta $\mathrm{x}$ cata) (Anexo 1), os quais foram reproduzidos por meio de figuras. Neste estudo foram analisados somente os pares de palavras que continham a estrutura CCV.

Os pares de palavras contidos na lista de palavras referida foram controlados no que se refere às variáveis linguísticas como: mesma tonicidade da sílaba (tônica ou átona); mesmo número de sílabas da palavra na forma de superfície (monossílabas, dissílabas e trissílabas); mesmo ambiente fonológico precedente e seguinte. Esse controle teve a finalidade de confirmar, com confiabilidade, que na ocorrência do AC, o mesmo foi resultado de um recurso para preencher foneticamente a posição silábica da segunda posição do OC e não consequência de interferência das variáveis linguísticas citadas.

Para identificar o uso da estratégia de AC, através da análise acústica, foi utilizado o software de áudio-processamento PRAAT, versão 4.6.09 (disponível em www.praat.org). Com a espectrografia foram medidos, em segundos (s), os tempos de emissão da vogal ou da fricativa, medidas isoladamente, em sílaba com o OC simplificado e o tempo de emissão da mesma vogal em uma sílaba com onset simples (OS). Nos casos de OC com fricativas, considerou-se o tempo de emis- são desta consoante, pois esta é a única classe das obstruintes que pode ser alongada, devido à presença do traço continuo na sua composição. Os tempos de emissão obtidos nas duas situações citadas foram comparados para verificar a presença ou ausência de AC. Considerou-se presente o AC quando a diferença entre os tempos de emissão isolados da vogal ou da fricativa em OC e OS foi maior ou igual a 0,04 s. Este valor foi estipulado com base em trabalhos do PB que investigaram a duração das vogais em diferentes contextos ${ }^{*}(9,10,21)$.

Foi considerada a porcentagem de $40 \%$ ou mais como uso produtivo da estratégia de $\mathrm{AC}$, fazendo-se uma analogia à aquisição dos fonemas no sistema fonológico proposto em outro estudo ${ }^{(22)}$. Na proposta do referido estudo, o fonema é considerado como adquirido quando sua ocorrência for de $80 \%$ a 100\%; parcialmente adquirido quando a ocorrência for de $40 \%$ a $79 \%$ e não adquirido quando sua ocorrência for igual ou inferior a 39\%. Assim, a realização de uma porcentagem igual ou superior a $40 \%$ de alongamento compensatório, seria indicativa de que esses sujeitos fazem uso produtivo da estratégia estudada.

$\mathrm{O}$ uso da estratégia de $\mathrm{AC}$ foi analisado em relação às diferentes gravidades do DF, conforme as classificações quantitativa e qualitativa. Os resultados obtidos foram submetidos a um estudo estatístico comparativo no qual foi utilizado o teste Qui-quadrado, considerando nível de significância de $\mathrm{p}<0,05$. Antes dos dados serem submetidos à análise estatística, excluiu-se da amostra o sujeito 21, pelo fato de ser o único a apresentar DS e DF com CIc. Sendo assim, a amostra submetida a tratamento estatístico foi composta de 20 sujeitos.

\section{RESULTADOS}

Na Figura 1 estão representados os resultados referentes à porcentagem de uso da estratégia de $\mathrm{AC}$ por gravidade, segundo a classificação quantitativa do DF. Observou-se relação positiva entre o percentual de uso da estratégia e a gravidade do $\mathrm{DF}$, ou seja, quanto maior a gravidade do DF, mais frequente o uso da estratégia. O estudo comparativo entre as gravidades, segundo a classificação quantitativa, no entanto, não mostrou diferença estatisticamente significativa entre os grupos (DM, DMM e DMS) $(\mathrm{p}>0,05)$.

Ao analisar a Figura 2, na qual estão representados os resultados da porcentagem de uso da estratégia de AC conforme a classificação qualitativa observou-se uma relação inversa. À medida que a gravidade do DF diminui, o uso da estratégia estudada aumenta. O estudo comparativo entre as gravidades, segundo a classificação qualitativa, no entanto, assim como na classificação quantitativa, não mostrou diferença estatisticamente significativa entre os grupos (CA e CI) $(p>0,05)$.

Através da observação da distribuição dos sujeitos conforme a gravidade do DF nas duas classificações utilizadas, verificou-se que os sujeitos classificados quantitativamente em DM, foram classificados qualitativamente como possuindo CA; os sujeitos classificados quantitativamente em DMM, foram classificados qualitativamente como possuindo CA e

* Faveri CB. Duração das vogais orais em português: um estudo preliminar. 2005 [manuscrito]. 


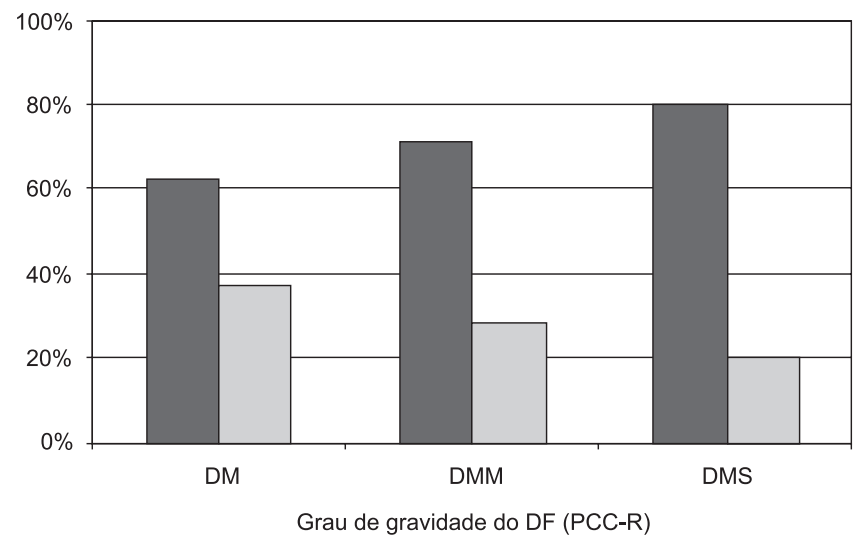

$\square \operatorname{Sim} \square$ Não

Legenda: $\mathrm{DF}=$ desvio fonológico; $\mathrm{PCC}-\mathrm{R}=$ Percentual de Consoantes Corretas - Revisado; $\mathrm{DM}$ = desvio fonológico de grau médio; $\mathrm{DMM}$ = desvio fonológico de grau médio-moderado; DMS = desvio fonológico de grau moderado-severo Teste Qui-quadrado: $\mathrm{p}=0,7949$

Figura 1. Porcentagens de uso da estratégia de alongamento compensatório $(A C)$ por gravidade, segundo a classificação quantitativa do desvio fonológico

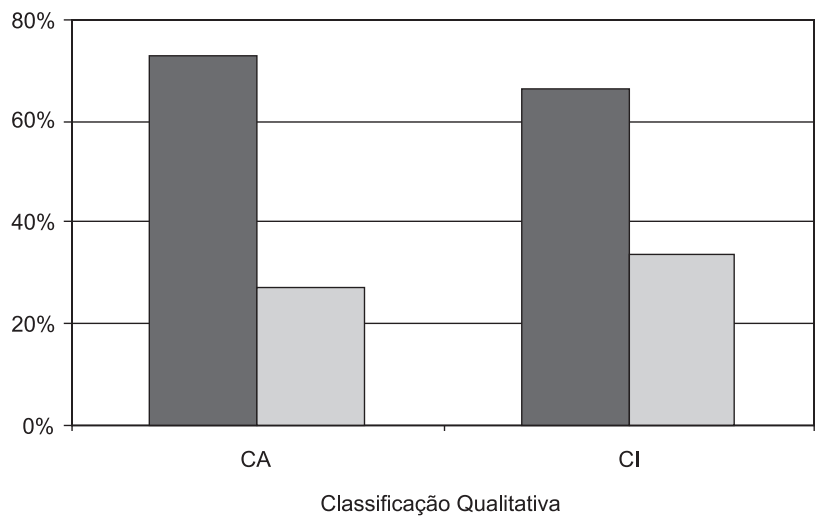

\section{$\square \operatorname{Sim} \square$ Não}

Legenda: $\mathrm{CA}$ = desvio fonológico com características atrasadas; $\mathrm{Cl}=$ desvio fonológico com características iniciais

Qui-quadrado: $p=0,7686$

Figura 2. Porcentagens de uso da estratégia de alongamento compensatório (AC), conforme a classificação qualitativa do desvio fonológico

CI e; os sujeitos que foram classificados quantitativamente em DMS, foram também classificados como possuindo CI, qualitativamente. Verificou-se correspondência entre as classificações quantitativa e qualitativa no que se refere à gravidade do DF (Tabela 1).

\section{DISCUSSÃO}

$\mathrm{Na}$ literatura especializada, tem-se observado interesse para se obter medidas objetivas que descrevam a produção de falantes com padrões fonológicos normais e desviantes, o que pode ser confirmado por meio de alguns estudos do $\mathrm{PB}^{(9-}$ 11,13,23). A importância de se estudar as propriedades acústicas dos sons da fala, se dá no sentido de que estas características possibilitam informações relevantes para o maior entendimento
Tabela 1. Distribuição dos sujeitos conforme a gravidade do DF nas duas classificações utilizadas

\begin{tabular}{lccccccc}
\hline Grupos & \multicolumn{6}{c}{ Classificação qualitativa } \\
& & \multicolumn{2}{c}{ CA } & Cl & \multicolumn{2}{c}{ Total } \\
& $\mathrm{N}$ & $\%$ & $\mathrm{~N}$ & $\%$ & $\mathrm{~N}$ & $\%$ \\
\hline $\begin{array}{l}\text { Classificação } \\
\text { quantitativa }\end{array}$ & $\mathrm{DM}$ & 8 & 40 & 0 & 0 & 8 & 40 \\
& & & & & & & \\
& DMM & 3 & 15 & 4 & 20 & 7 & 35 \\
Total & DMS & 0 & 0 & 5 & 25 & 5 & 25 \\
\hline & & 11 & 55 & 9 & 45 & 20 & 100 \\
\hline
\end{tabular}

Legenda: $\mathrm{CA}$ = desvio fonológico com características atrasadas; $\mathrm{Cl}=$ desvio fonológico com características iniciais; $\mathrm{DM}$ = desvio fonológico de grau médio; $\mathrm{DMM}$ = desvio fonológico de grau médio-moderado; $\mathrm{DMS}$ = desvio fonológico de grau moderado-severo

sobre o funcionamento articulatório e da fonologia do sujeito, o que consequentemente auxiliaria tanto no conhecimento sobre aquisição fonológica normal, como no diagnóstico, tratamento e prognóstico dos casos atípicos ${ }^{(24)}$. Com base em estudos citados anteriormente, que utilizaram análise acústica em dados de fala, empregou-se esta ferramenta no intuito de se obter maior fidedignidade e precisão na identificação da estratégia de $\mathrm{AC}$ - nem sempre identificada perceptivamente - na fala de crianças com diagnóstico de DF que realizam a estratégia de simplificação de OC.

Neste estudo, a simplificação do OC esteve presente em todos os sujeitos, independente da gravidade do DF, de acordo com a análise perceptivo-auditiva realizada. Outros autores verificaram que a simplificação do OC aumenta à medida que a severidade do desvio diminui ${ }^{(25)}$. Este fato ocorre porque a simplificação do OC é uma estratégia que desaparece tardiamente da fala das crianças ${ }^{(5,6)}$.

Ao analisar a ocorrência do uso da estratégia de AC segundo as gravidades do DF propostas na classificação quantitativa, verificou-se que a porcentagem do uso da estratégia foi semelhante entre as mesmas, sem diferença estatisticamente significativa entre eles.

Os resultados desta pesquisa mostram que, mesmo sem diferença estatisticamente significativa, na classificação quantitativa, à medida que a gravidade do DF diminui, o uso da estratégia de AC também o faz, contrariando a hipótese inicial deste estudo. Acreditava-se que a estratégia de AC aumentaria com a diminuição da gravidade do DF, uma vez que tal estratégia representa um conhecimento mais sofisticado em relação à produção da criança. Por outro lado, esses resultados vão ao encontro de outro achado da literatura ${ }^{(25)}$, o qual refere que o número de processos fonológicos diminui à medida que $\mathrm{a}$ gravidade do desvio também diminui. Este achado aplica-se à estratégia de AC independentemente de esta ser um processo fonológico mais ou menos sofisticado.

Nesta pesquisa a análise do uso da estratégia de $\mathrm{AC}$ e da gravidade do DF, segundo a classificação qualitativa, mostrou que mesmo sem significância estatística, quanto menor a gravidade do DF, mais frequente o uso da estratégia estudada. Justifica-se este resultado pelo fato de que à medida que a gravidade do DF diminui, supõe-se que haja maior conhecimento fonológico por parte destes sujeitos, o que explica a 
utilização produtiva da estratégia de $\mathrm{AC}^{(9-11)}$.

A construção do conhecimento fonológico se dá à medida que os traços fonológicos vão sendo adquiridos e organizados em uma hierarquia. Alterações, sejam ligações ou desligamentos entre os traços, vão ser observadas na fala dos sujeitos como erros e, também, como indícios de construção do sistema fonológico ${ }^{(26,27)}$. Fazendo uma analogia da aquisição segmental ao domínio das estruturas silábicas, também se pode conceber como conhecimento fonológico a disponibilidade de um constituinte prosódico, verificado pelo seu preenchimento correto (ex.: parto - ['partu]) ou inadequado (ex.: parto - ['pajtu]) ou pelo alongamento compensatório de um segmento adjacente $(\text { ex.: parto - [pa:tu] })^{(11)}$.

$\mathrm{Na}$ pesquisa em que foi proposta a tipologia qualitativa do DF, utilizada neste trabalho, a autora investigou a relação dessa tipologia com a gravidade do desvio pelo PCC - classificação quantitativa do $\mathrm{DF}^{(20)}$. A autora observou que o grupo que apresenta DF com CIc tem representantes de sujeitos principalmente com DS, DMS, e em menor número, DMM. $\mathrm{O}$ grupo com $\mathrm{CI}$ tem representantes de sujeitos principalmente com DMS e DMM. Há sujeitos com DMM e, principalmente, DM no grupo com CA. No grupo que a autora classificou como possuindo CFA, há sujeitos com todos os graus de gravidade citados anteriormente.

Os resultados da presente pesquisa mostram que há correspondência entre a gravidade do DF, classificado quantitativa e qualitativamente, ou seja, sujeitos que foram classificados com uma maior gravidade do desvio também foram classificados qualitativamente como possuindo uma fonologia menos sofisticada. Estes dados são concordantes com os de outros autores ${ }^{(20,28)}$.

Em um estudo ${ }^{(29)}$ que relacionou a gravidade do DF e tipologia do DF - com base em outras já propostas ${ }^{(20,25)}$, as autoras encontraram a mesma correspondência já citada entre as classificações quantitativa e qualitativa do DF.

Nesta pesquisa observou-se que a classificação qualitativa do DF parece ser mais adequada para descrever o uso da estratégia de AC, apesar de não considerá-la para sua composição. Com esta classificação pode-se perceber detalhes do conhecimento fonológico dos sujeitos, já que a mesma faz distinção entre processos de estrutura silábica e processos envolvendo segmentos (fonemas). Dentro dos processos de estrutura silábica poderiam estar alocados os casos de AC, evidenciando conhecimento subjacente do constituinte OC. Da mesma forma, a análise qualitativa consegue fazer distinção entre tipos de processos e o que eles representam em termos de conhecimento fonológico. Por exemplo, pela fonologia gerativa não linear o processo de semivocalização (ex.: caro
- ['karu] $\rightarrow$ ['kaju]; não ligação dos traços de Ponto de Consoantes, ou seja, [coronal, +anterior] e [+contínuo]) mostra menor conhecimento da criança em relação a um processo de dessonorização (ex.: bato - ['batu] $\rightarrow$ ['patu]), no qual apenas a definição do valor do traço sonoro está alterada. O PCC-R não prevê esta distinção entre tipos de processos nos seus graus de classificação. Os achados referentes à análise qualitativa confirmam a hipótese inicial que motivou este estudo, de que quanto maior o uso de AC, maior o conhecimento fonológico e, portanto, menor é o grau do desvio de fala. Isso reforça que as duas classificações devem ser utilizadas na clínica fonoaudiológica, pois são complementares e nos possibilitam analisar a fala dos sujeitos a partir de diferentes perspectivas.

Alguns autores reconhecem a importância da utilização concomitante de classificações quantitativas e qualitativas, pois, identificando-se o tipo e o grau de gravidade do DF, o fonoaudiólogo pode determinar a real situação do sistema fonológico do sujeito, bem como refletir sobre decisões terapêuticas ${ }^{(20,25,28)}$. Por outro lado, quando nos referimos a estratégia de AC, a classificação qualitativa mostra-se mais eficiente, como já discutido anteriormente.

Estudos sobre a estratégia de AC e sua relação com DF e suas classificações são escassos, por isso, ressalta-se a necessidade de pesquisas adicionais sobre este assunto com o auxílio da análise acústica que adicionará objetividade à descrição do sistema fonológico do sujeito.

O presente estudo fornece não somente um benefício teórico descritivo referente à caracterização dos DF, como também contribui para a clínica fonoaudiológica, representando um instrumento de descrição fonológica objetiva, por meio da análise acústica para interpretação dos dados de fala dos sujeitos, antes, durante e após o processo terapêutico. Da mesma forma, fornece informações acerca da estratégia de AC pouco citada na literatura especializada até o momento, mostrando-a como mais um recurso utilizado pelo sujeito, para compensar sua produção alterada.

\section{CONCLUSÃO}

A partir deste estudo constatou-se que não há relação entre o uso da estratégia de AC e as diferentes gravidades do DF. O uso da estratégia estudada foi semelhante nas diferentes gravidades do DF em ambas as classificações e sem diferença estatisticamente significante nos estudos comparativos realizados.

A distribuição dos sujeitos por gravidade do DF segundo as classificações quantitativa e qualitativa permitiu visualizar uma correspondência entre as gravidades utilizadas nas duas classificações.

\begin{abstract}
Purpose: To verify the existence of a relationship between the compensatory lengthening strategy and the different severity levels of phonological disorder, quantitatively and qualitatively classified, as well as to verify the existence of a correspondence between these severity classifications. Methods: Twenty children with different severity levels of phonological disorder and ages ranging from five to ten years participated in the study. Phonological disorder was quantitatively and qualitatively classified for each subject. The use of the compensatory lengthening strategy was identified through acoustic analysis, using an audio-processing software (PRAAT). The collected data were statistically analyzed using the Chi-square test. Results: The comparative study of the severity levels of
\end{abstract}




\begin{abstract}
phonological disorder, according to both quantitative and qualitative classifications, did not show statistically significant differences among the groups, regarding the compensatory lengthening strategy. A descriptive analysis of the subjects' distribution according to the severity of the phonological disorder in both classifications showed a correspondence between quantitative and qualitative classifications regarding the severity of the phonological disorder. Conclusion: There was no relationship between the use of the compensatory lengthening strategy and the different phonological disorder severities. Subjects' distribution based on the severity of the phonological disorder, according to the classifications used in the study, allowed the visualization of a correspondence between both classifications of the disorder.
\end{abstract}

Keywords: Speech disorders; Speech acoustics; Speech; Sound spectrography/analysis; Speech-language pathology; Child language

\title{
REFERÊNCIAS
}

1. Lamprecht RR. (Org.). Aquisição fonológica do português: perfil de desenvolvimento e subsídios para terapia. Porto Alegre: Artmed; 2004. Sobre os desvios fonológicos. p. 193-212.

2. Ribas LP. Onset complexo: características da aquisição. Letras Hoje. 2003;38(2):23-31.

3. Wertzner HF, Sotelo MB, Amaro L. Analysis of distortions in children with and without phonological disorders. Clinics (São Paulo). 2005;60(2):93-102.

4. Wertzner HF, Galea DES. Porcentagem de consoantes corretas revisada (PCC-R) e índice de densidade fonológica (PDI) na aquisição fonológica. Rev Soc Bras Fonoaudiol. 2002;7(1):44-50.

5. Lamprecht RR. A aquisição da fonologia do português na faixa etária dos 2:9 - 5:5. Letras Hoje. 1993;28(2):99-106.

6. Othero GA. Processos fonológicos na aquisição da linguagem pela criança. ReVEL. 2005;3(5):1-13.

7. Patah LK, Takiuchi N. Prevalência das alterações fonológicas e uso dos processos fonológicos em escolares aos 7 anos. Rev CEFAC. 2008;10(2):158-67.

8. Miranda ICC. Aquisição e variação estruturada de encontros consonantais tautossilábicos [tese]. Belo Horizonte(MG): Faculdade de Letras, Universidade Federal de Minas Gerais; 2007.

9. Miranda AR. Evidências acústicas sobre a fixação do parâmetro da coda no português brasileiro. In: Hernandorena CLM. Aquisição de língua materna e de língua estrangeira: aspectos fonéticos-fonológicos. Pelotas: EDUCAT; 2001. p. 145-58.

10. Mezzomo CL. A análise acústica como subsídio para a descrição da aquisição do constituinte coda. Letras Hoje. 2003;38(2):75-82.

11. Mezzomo CL. O uso de estratégia de reparo como indício do conhecimento fonológico da criança. In: Bonilha GFG, Keske-Soares M. Estudos em aquisição fonológica. Santa Maria: PPGL Editores; 2007. p. 65-80.

12. Goulart BNG, Chiari BM. Prevalência de desordens de fala em escolares e fatores associados. Rev Saúde Pública. 2007;41(5):726-31.

13. Pagan LO, Wertzner HF. Análise acústica das consoantes líquidas do Português Brasileiro em crianças com e sem transtorno fonológico. Rev Soc Bras Fonoaudiol. 2007;12(2):106-13.

14. McLeod S, Isaac K. Use of spectrographic analyses to evaluate the efficacy of phonological intervention. Clin Linguist Phon. 1995;9(3):229-34.

15. Maxwell E, Weismer G. The contribution of phonological, acoustic, and perceptual techniques to the characterization of a misarticulating child's voice contrast for stops. Appl Psycholinguist. 1982;3:29-43.
16. Yavas M, Hernandorena CLM, Lamprecht RR. Avaliação fonológica da criança: reeducação e terapia. Porto Alegre: Artes Médicas; 1991.

17. Wertzner HF, Amaro L, Galea DES. Phonological performance measured by speech severity indices compared with correlated factors. São Paulo Med J. 2007;125(6):309-14.

18. Shriberg LD, Austin D, Lewis BA, McSweeny JL, Wilson DL. The percentage of consonants correct (PCC) metric: extensions and reliability data. J Speech Lang Hear Res. 1997;40(4):708-22.

19. Shriberg LD, Kwiatkowski J. Phonological disorders I: a diagnostic classification system. J Speech Hear Disord. 1982;47(3):226-41.

20. Keske-Soares M. Terapia fonoaudiológica fundamentada na hierarquia implicacional dos traços distintivos aplicada em crianças com desvios fonológicos [tese]. Porto Alegre(RS): Faculdade de Letras, Pontifícia Universidade Católica do Rio Grande do Sul; 2001.

21. Gama-Rossi A. Relações entre percepção e produção na aquisição da duração da vogal no português brasileiro. Letras Hoje. 2001;36(3):17786.

22. Bernhardt B. Developmental implications of nonlinear phonological theory. Clin Linguist Phon. 1992;6(4):259-81.

23. Wertzner HF, Pagan-Neves LO, Castro MM. Análise acústica e índice de estimulabilidade nos sons líquidos do português brasileiro. Rev CEFAC. 2007;9(3):339-50.

24. Bonatto MTRL. A produção de plosivas por crianças de três anos falantes do português brasileiro. Rev CEFAC. 2007;9(2):199-206.

25. Keske-Soares M, Blanco APF, Mota HB. O desvio fonológico caracterizado por índices de substituição e omissão. Rev Soc Bras Fonoaudiol. 2004;9(1):10-18.

26. Hernandorena CLM. Sobre a descrição de desvios fonológicos e de fenômenos da aquisição da fonologia. Letras Hoje. 1995;30(4):91-110.

27. Hernandorena CLM. Relações implicacionais na aquisição da fonologia. Letras Hoje. 1996;31(2):67-76.

28. Mota HB, Dias RF, Ghisleni MRL, Keske-Soares M, Mezzomo CL. A análise do desvio fonológico evolutivo utilizando-se abordagem quantitativa e qualitativa. Anais do $16^{\circ}$ Congresso Brasileiro de Fonoaudiologia; 2008 Set 24-27; Campos do Jordão: Sociedade Brasileira de Fonoaudiologia; 2008. Rev Soc Bras Fonoaudiol. 2008;Supl Espec:561.

29. Vieira MG, Mota HB, Keske-Soares M, Dias RF. Classificação quantitativa versus qualitativa do grau de gravidade dos desvios fonológicos. Anais do $16^{\circ}$ Congresso Brasileiro de Fonoaudiologia. 2008 Set 24-27; Campos do Jordão: Sociedade Brasileira de Fonoaudiologia; 2008. Rev Soc Bras Fonoaudiol. 2008;Supl Espec:561. 
Anexo 1. Lista de palavras utilizada para a análise da estratégia de alongamento compensatório (AC)

\begin{tabular}{|c|c|}
\hline \multicolumn{2}{|c|}{ Onset complexo } \\
\hline 1. Preso - Peso & 21. Trapo - Tapo \\
\hline 2. Prego - Pego & 22. Traça - Taça \\
\hline 3. Pregar - Pegar & 23. Contra - Conta \\
\hline 4. Pressa - Peça & 24. Trem - Tem \\
\hline 5. Prata - Pata & 25. Troca - Toca \\
\hline 6. Praça - Passa (roupa) & 26. Frio - Fio \\
\hline 7. Praga - Paga & 27. Drama - Dama \\
\hline 8. Prato - Pato & 28. Cravada - Cavada \\
\hline 9. Prisão - Pisão & 29. Craque - Caqui \\
\hline 10. Pronto - Ponto & 30. Crosta - Costa \\
\hline 11. Templo - Tempo & 31. Classe - Cace \\
\hline 12. Plano - Pano & 32. Grama - Gama \\
\hline 13. Pluma - Puma & 33. Magro - Mago \\
\hline 14. Branco - Banco & 34. Frase - Fase \\
\hline 15. Branca - Banca (de revista) & 35. Lavrador - Lavador \\
\hline 16. Brabo - babo & 36. Flauta - Falta \\
\hline 17. Broa - Boa & 37. Flecha - Fecha \\
\hline 18. Brota - Bota & 38. Floco - Foco (de luz) \\
\hline 19. Bruxa - Bucha & 39. Flora (nome) - Fora \\
\hline 20. Cravo - Cavo & 40. Centro - Sento \\
\hline \multicolumn{2}{|c|}{ Coda } \\
\hline 1. Calçou - Caçou & 16. Prisma - Prima \\
\hline 2. Falcão - Facão & 17. Par - pá \\
\hline 3. Solzinho - Sozinho & 18. Cruz - Cru \\
\hline 4. Polvo - Povo & 19. Voz - Vó \\
\hline 5. Volta - Vota & 20. Nós - Nó \\
\hline 6. Caldeira - Cadeira & 21. Certa - Seta \\
\hline 7. Talco - Taco & 22. Barba - Baba \\
\hline 8. Sol-Só & 23. Largo - Lago \\
\hline 9. Fácil - Face & 24. Porte - Pote \\
\hline 10. Costas - Cotas & 25. Cerca - Seca \\
\hline 11. Poste - Pote & 26. Carta - Cata \\
\hline 12. Pasto - Pato & 27. Marcho - Macho \\
\hline 13. Pastinho - Patinho & 28. Farto - Fato \\
\hline 14. Risca - Rica & 29. Gasto - Gato \\
\hline 15. Masca - Maca & \\
\hline
\end{tabular}

